\title{
CHANCE CONSTRAINED DATA ENVELOPMENT ANALYSIS FOR EFFICIENCY ANALYSIS: AN APPLICATION TO TURKISH MANUFACTURE OF IRON AND STEEL SECTOR
}

\author{
Hakan Gedik ${ }^{1, *}$, Hasan Bal $^{2}$ and Mahmut İzciler ${ }^{3}$ \\ ${ }^{1}$ Department of Computer Education, Gazi University, 06830, Gölbaş1, Ankara, Turkey \\ ${ }^{2}$ Department of Statistics, Gazi University, 06500, Teknikokullar, Ankara, Turkey \\ ${ }^{3}$ Department of Industrial Technology Education, Gazi University, 06830, Gölbaş1, \\ Ankara, Turkey \\ hgedik@gazi.edu.tr, hasanbal@gazi.edu.tr, mizciler@gazi.edu.tr
}

\begin{abstract}
This study aims to determine the technical efficiency levels of the enterprises active in the "Manufacture of Basic Iron and Steel and of Ferro-Alloys" sector in Turkey. The inputs and outputs are deterministic in classical Data Envelopment Analysis, so the changes in exchange rate, inflation rate, etc. aren't considered, and the precautions for future inconsistencies are not foreseen. This leads to critics of deterministic Data Envelopment Analysis models. In this paper, the additive model developed depending on the Banker, Charnes and Cooper (BCC) model was extended by chance constrained programming formulations in order to overcome the insufficiencies in deterministic Data Envelopment Analysis, and the technical analysis of "Manufacture of Basic Iron and Steel and of Ferro-Alloys" sector was performed.
\end{abstract}

Key Words- Efficiency, data envelopment analysis, chance constrained programming

\section{INTRODUCTION}

Data Envelopment Analysis (DEA) is a technique used in the evaluation of the efficiencies of decision making units (DMUs) that use multiple inputs for producing multiple outputs. Up to now since it has appeared, DEA has found widespread applications in various areas and still goes on its advancement with an interaction to many techniques. In fact, DEA is the most popular approximation which developed very fast and has widespread applications especially in the operations research and other science areas. In the study the micro-level data pertaining to the mentioned sector which have been compiled by the Turkish Statistical Institute (TurkStat) have been evaluated, and it should also be noted that these data are the most recently published annual data. Using the micro-data of the production units for the year 2004, the technical efficiency of the Manufacture of Basic Iron and Steel and of Ferro-Alloys" sector was analyzed according to the deterministic DEA BCC Additive model and Chance Constrained DEA (CCDEA) Additive model.

The contribution of the study is two dimensional; under the light of previous scientific studies conducted, using micro-data on the basis of production rather than panel data and applying the deterministic DEA and CCDEA method, the measurement of technical efficiency was studied and the results were compared. On the other hand, by applying the methodology to the "Manufacture of Basic Iron and Steel and of Ferro- 
Alloys" sector, technical efficiency of the sector was measured. The layout of the article is as follows: in the second part basic DEA approach is presented, in the third part Additive DEA models are explained. In the fourth part, the chance constrained DEA models are presented. In the fifth part, determination of input and output variables are presented, and in the sixth part, a real data application and the results of this application entirely are discussed and interpreted.

\section{BASIC APPROACHES IN DEA}

Following the first study concerning the production efficiency by Farrell [1], numerous methods were used regarding the measurement of efficiency. The most widely employed methods for setting an efficiency margin are the econometrical stochastic frontier approach (SFA), and the data envelopment analysis, which is a mathematical programming method.

SFA is based on an econometrical model and it requires the determination of a functional structure. Thus SFA is found to be limiting, yet the SFA method is preferred in many studies for its separation of statistical errors from errors caused by inefficiency [2].

Data envelopment analysis is used frequently in the sectors where different input and output units are utilized, since it is based on the production technology. In contrast to the econometrical methods, DEA is widely used because it does not require functional structures such as the production function, cost function etc.

The main forms of DEA models and their extensions include the CCR model [3], the BCC model [4] and the additive models [5]. In these treatments, the input and output data are always assumed to be deterministic but the surveys in Lovell [6] and Banker and Cooper [7] extend this to the introduction of chance (statistical or probabilistic) elements in DEA [8].

This classification can be further divided into three on the basis of orientation as non-oriented, input-oriented, and output-oriented. The oriented models in the data envelopment analysis can be named as input reduction and output augmentation models. The objective of the former is how to reduce the inputs to obtain the given level of output, while the objective of the latter is to obtain the highest possible level of output with a given input level [9].

The BCC model had been proposed by Banker, Charnes and Cooper. The BCC model measures the efficiency of decision making units under the assumption of variable return-to-scale. Hence, pure technical efficiency can be obtained with the BCC model under the assumption of variable return-to-scale.

\section{BCC-ADDITIVE MODEL}

Basic approaches such as CCR and BCC models conduct separate evaluations concerning input and output. But in the additive model there is no input-output orientation, the model evaluates these two types of orientation together. Additive model was developed in 1985 by Charnes et al [5]. 
In this paper, the "return-to-scale" assumption of BCC model is included into the additive model in order to develop BCC-Additive model, and its formulation is as follows;

\section{BCC-Additive Model}

$\max \sum_{\mathrm{r}=1}^{\mathrm{s}} \mathrm{s}_{\mathrm{r}}^{+}+\sum_{\mathrm{i}=1}^{\mathrm{m}} \mathrm{s}_{\mathrm{i}}^{-}$

Constraints:

$$
\begin{aligned}
& \sum_{j=1}^{n} x_{i j} \lambda_{j}+s_{i}^{-}=x_{i 0} \forall i \\
& \sum_{j=1}^{n} y_{r j} \lambda_{j}-s_{r}^{+}=y_{r 0} \forall r \\
& s_{r}^{+}, s_{i}^{-}, \lambda_{j} \geq 0, \forall r, i, j \\
& \sum \lambda_{j}=1
\end{aligned}
$$

where

$i=1, \ldots, m$ inputs

$r=1, \ldots, s$ outputs

$j=1, \ldots, n$ the collection of DMUs

$s^{+}=s_{r}^{+} \quad$ and $\quad s^{-}=s_{i}^{-}$are column vectors of slack variables

$x_{i j}=$ sample input matrix

$y_{r j}=$ sample output matrix

$x_{i 0}=$ column vector of inputs of the particular DMU investigated

$y_{r 0}=$ column vector of outputs of the particular DMU investigated

$\lambda=$ weight of input and output

The total of $\lambda$ 's is equal to 1 in model.

The actual purpose here is to calculate the farthest point to the inefficient decision unit on the efficiency margin, assessing the excess input $s_{i}^{-}$and output deficit $s_{r}^{+}$together. The efficiency level can not be obtained at the end of this model, however for the decision units, being efficient or not can be assessed on the basis of the values of the slack variables, and it can also be stated that there is no Pareto-Koopmans efficiency [5].

If both idle variables are found to be $s_{i}^{-}=0$ and $s_{r}^{+}=0$, that decision making unit is deemed to be efficient according to the additive model. If any one or both of the slack variables are not zero, it is stated that the total of the values of the non-zero ones defines the amount of inefficiency in inputs and outputs.

In brief, the additive model is a model which is based on the variable return-toscale, and which associates data envelopment with inefficiency analysis of CharnesCooper. 


\section{CHANCE CONSTRAINED MODEL DERIVED FROM BCC-ADDITIVE}

The main critics about DEA are that the inputs and the outputs are assumed to be deterministic in this method. An efficient DMU can be inefficient in case of the presence of a random variable. Studies on CCDEA have been started and continuing in order to eliminate the problems arising because of randomness.

The chance constrained programming models have first been introduced by Charnes et al. [9]. The first CCDEA model was developed by Land et al. [10]. Sengupta [11-13], Land et al. [14], Land et al. [15], Olesen and Petersen [16], Olesen and Petersen [17], Cooper et al. [18], Li [19] etc. have conducted studies on CCDEA. The LLT model was developed by Land et al. [14], and the OP model was developed by Olesen and Petersen [16]. Regarding all the studies performed, the BCC-Additive model has been shifted from being deterministic to chance constrained in this paper.

Hereafter, the CCBCC-Additive model derived from BCC-Additive model is illustrated. The covariance between inputs and outputs is ignored.

$$
\max \sum_{r=1}^{s} s_{r}^{+}+\sum_{i=1}^{m} s_{i}^{-}
$$

Constraints:

$$
\begin{aligned}
& P\left\{\sum_{j=1}^{n} y_{r j} \lambda_{j} \geq y_{r 0}\right\} \geq 1-\alpha \\
& r=1, \ldots, s \\
& P\left\{\sum_{j=1}^{n} x_{i j} \lambda_{j} \leq x_{i 0}\right\} \geq 1-\alpha \\
& i=1, \ldots, m \\
& \sum_{j=1}^{n} \lambda_{j}=1 \\
& \lambda_{j} \geq 0, j=1, \ldots, n \\
& \lambda=\left[\lambda_{j}\right] \text { is the weighted column vector determining the best application of the }
\end{aligned}
$$

evaluated DMU. 1- $\alpha$ shows the probability of observed outputs not to be higher than the maximum output, or observed inputs not to be less than the minimum input.

Land et al. [14] assumes that inputs and outputs are random, and outputs show cumulative normal distribution depending on the inputs in order to reach CCDEA. The following transformations occur when the output constraint is considered in model 2 :

$$
P\left\{\sum_{j=1}^{n} y_{r j} \lambda_{j} \geq y_{k}\right\} \geq 1-\alpha
$$

Now let's define a new variable

$$
u=\sum_{j=1}^{n} y_{k} \lambda_{j}-y_{k}
$$

Then; 


$$
\begin{aligned}
& E(u)=\sum_{j=1}^{n} \mu_{j} \lambda_{j}-y_{k}=\mu_{u} \\
& \operatorname{Var}(\mathrm{u})=\sum_{j=1}^{n} \sigma_{j}^{2} \lambda_{j}^{2}+\left(\lambda_{0}-1\right)^{2} \sigma_{k}^{2}=\sigma_{u}^{2}
\end{aligned}
$$

$u$ will also have the same distribution with $y$.

$$
\mathrm{z}=\frac{\mathrm{u}-\mu_{\mathrm{u}}}{\sigma_{\mathrm{u}}}
$$

As normal distribution is symmetrical, it becomes

$$
\mathrm{P}\left\{\sum_{j=1}^{n} y_{j} \lambda_{j} \geq y_{k}\right\}=\mathrm{P}\{u \geq 0\}=\mathrm{P}\left\{Z \geq-\frac{\mu_{u}}{\sigma_{u}}\right\}=\Phi\left(\frac{\mu_{u}}{\sigma_{u}}\right)
$$

$\Phi$ is the standard normal distribution function.

$$
\Phi\left(\frac{\mu_{u}}{\sigma_{u}}\right) \geq(1-\alpha)
$$

Different envelopments of the data are obtained through assigning different values between 0 and 1 for $\alpha$.

$\mu \geq \Phi^{-1}(\alpha) \sigma_{u}$ in Eqn. 8 becomes Eqn. 9 in chance constrained DEA model.

$$
\sum_{j=1}^{n} \lambda_{j} \mu_{k} \geq \mu_{k}+\Phi^{-1}(\alpha) \sqrt{\sum_{\substack{j=1 \\ j \neq k}}^{n} \lambda_{j}^{2} \sigma_{j}^{2}+\left(\lambda_{k}-1\right)^{2} \sigma_{k}^{2}}
$$

Regarding the assumption by Land et al. (1993) in order to reach CCCDEA model, $\operatorname{cov}\left(y_{i,} y_{j}\right)=\sigma_{i j}=0$. Then $\sigma_{j}^{2}=\sigma^{2}$.

At the end, Eqn. 10 is achieved;

$$
\sum_{j=1}^{n} \lambda_{j} \mu_{j}-\Phi^{-1}(\alpha) \sigma \sqrt{\sum_{j=1}^{n} \lambda_{j}^{2}+\left(\lambda_{k}-1\right)^{2}} \geq y_{k}
$$

The following transformations are realized in input constraint as it is in output constraint.

$$
P\left\{\sum_{j=1}^{n} x_{i j} \lambda_{j} \leq x_{k}\right\} \geq 1-\alpha
$$

Now let's define a new random variable;

$$
v=\sum_{J=1}^{n} x_{i j} \lambda_{j}-x_{k}
$$

And according to this variable;

$$
\begin{aligned}
& E(v)=\sum_{j=1}^{n} \mu_{j} \lambda_{j}-x_{k}=\mu_{v} \\
& \operatorname{Var}(v)=\sum_{j=1}^{n} \sigma_{i j}^{2} \lambda_{j}^{2}+\left(\lambda_{0}-1\right)^{2} \sigma_{k}^{2}=\sigma_{u}^{2}
\end{aligned}
$$

$v$ has the same distribution with $x$. 


$$
Z=\frac{v-\mu_{v}}{\sigma_{v}}
$$

Then;

$$
p\left\{\sum_{j=1}^{n} x_{k} \lambda_{j} \leq x_{k}\right\}=\operatorname{Pr}\{u \leq 0\}=\operatorname{Pr}\left\{Z \leq-\frac{\mu \nu}{\sigma_{v}}\right\}
$$

And as normal distribution has symmetrical features, Eqn. 15 is reached.

$$
\operatorname{Pr}\left\{Z \leq-\frac{\mu_{v}}{\sigma_{v}}\right\}=-\Phi\left(\frac{\mu_{v}}{\sigma_{v}}\right)
$$

Then;

$$
-\Phi\left(\frac{\mu_{v}}{\sigma_{v}}\right) \geq 1-\alpha
$$

Different envelopments of the data are obtained through assigning different values between 0 and 1 for $\alpha$.

$$
\begin{aligned}
& \mu_{v} \geq-\left(\Phi^{-1}(\alpha) \sigma_{v}\right) \\
& \sum_{j=1}^{n} \lambda_{j} \mu_{j}-\mu_{k} \geq \Phi^{-1}(\alpha) \sqrt{\sum_{j=1}^{n} \lambda_{j}^{2} \sigma_{j}^{2}\left(\lambda_{0}-1\right)^{2} \sigma_{k}^{2}}
\end{aligned}
$$

Then the input constraint in chance constrained DEA will have the form in Eqn. 19.

$$
\sum_{j=1}^{n} \lambda_{j} \mu_{j} \geq \mu_{k}-\Phi^{-1}(\alpha) \sigma \sqrt{\sum_{\substack{j=1 \\ j \neq k}}^{n} \lambda_{j}^{2}+\left(\lambda_{k}-1\right)^{2}}
$$

At the end Eqn. 20 will be reached.

$$
\sum_{j=1}^{n} \lambda_{j} \mu_{j}-\Phi^{-1}(\alpha) \sigma \sqrt{\sum_{J=1}^{n} \lambda_{j}^{2}-2 \lambda_{k}+1} \geq x_{k}
$$

Eqn. 21 shows the deterministic equation of the model derived from the BCC-Additive model using formulations between Eqn. 4 and Eqn. 20

$$
\begin{aligned}
& \max \sum_{r=1}^{s} s_{r}^{+}+\sum_{i=1}^{m} s_{i}^{-} \\
& \text {klsitlar: } \\
& \sum_{j=1}^{n} \lambda_{j} x_{i j}+s_{i}^{-}-\Phi^{-1}(\alpha) \sigma \sqrt{\sum_{J=1}^{n} \lambda_{j}^{2}-2 \lambda_{k}+1}=x_{i o} \\
& \sum_{j=1}^{n} \lambda_{j} y_{r j}-s_{r}^{+}+\Phi^{-1}(\alpha) \sigma \sqrt{\sum_{J=1}^{n} \lambda_{j}^{2}+2 \lambda_{k}+1}=y_{r o} \\
& \sum_{j=1}^{n} \lambda_{j}=1 \\
& \lambda_{j} \geq 0, s_{r}^{+} \geq 0, s_{i}^{-} \geq 0, j=1, \ldots, n \\
& r=1, \ldots, s ; i=1, \ldots, m
\end{aligned}
$$

$\Phi$ is the standard normal distribution function and $\Phi^{-1}(\alpha)$ denotes the values from "standard normal cumulative probability" table. 
The operator $\sigma$ is the standard deviation. $\Phi^{-1}\left(\alpha_{i}\right)$ is equal to 0 in case $\alpha_{i}=0,50$. This independence assumption gives rise to the diagonal matrix with zero off-diagonal elements [14].

For the level of $\alpha$ other than 0.50 , the effect of the chance constraint moves the envelope. That is, it does not lie within the constraint belonging to the valid subset (in the envelop), but is located at a certain distance from these points. Chance constraints do not provide confidence intervals around the mean. $\alpha$ can be set at different levels $(0.01,0.05$, etc. $)$.

Another information obtained in CCDEA models, in contrast to other methods, is the level of output buffer and input buffer pertaining to DMUs which were found to be inefficient.

In other words, it guarantees that the possibility of observed outputs exceeding best outputs is equal to or lower than a threshold level [20].

The stochastic structure of the CCDEA model allows setting a more flexible production frontier when compared to DEA. On the other hand, the findings obtained through both methods follow a matching path. The CCDEA structure described above can be applied to all DEA approaches.

\section{DETERMINATION OF INPUT AND OUTPUT VARIABLES}

In the study the micro-data compiled by TurkStat from enterprises belonging to the sub-sector of "Manufacture of Basic Iron and Steel and of Ferro-Alloys" (NACE Rev. $1.1^{1}$ - 27.10) under the "Primary metal industry" sector (NACE Rev. 1.1 - 27) through the "Structural Business Statistics Enterprise Survey" are considered. The data refers to 2004 as it is the last available and published data by TurkStat. The inputs and the outputs depending on the available data are as follows:

Inputs (in Turkish Lira - TL)

- Manufacture costs: Value of equipment, raw and auxiliary materials purchased to be used in production of goods and services

- Employment costs: Total personnel cost divided by the number of employees

- Other costs: Value of goods purchased to be sold without further processing, rent expenditure of the enterprise, rent expenditure of machinery and equipment, payments made to subcontracted firms, financial expenditures

- Energy costs: Purchase of electricity, expenditure of fuels (heat, steam, hot water, natural gas) used during the production process

Outputs (in Turkish Lira - TL)

- Production revenues: Income from sales of manufacturing industry's production

- Other revenues: Income from wholesale trade, retail trade, service activities, rent income, financial income

- The value of the exports: Export value of goods

\footnotetext{
${ }^{1}$ NACE Rev.1.1: Statistical Classification of Economic Activities in the European Community, Revision 1.1
} 


\section{APPLICATION}

Firstly, the deterministic analysis using Eqn. (1) was performed considering the data pertaining to inputs and outputs. Then, a new analysis was performed using Eqn. (21) for $\alpha=0.05$ in order to make it possible to apply chance constrained DEA model.

54 firms meeting the DEA requirements for the sector were covered in the analysis. The data obtained was analyzed using the program LINGO 11.

Table 1. Summary statistics per Input and Output variables concerning 54 DMUs

\begin{tabular}{|l|l|l|l|l|}
\hline VARIABLES (in TL) & Std. Dev. & Mean & Min. & Max. \\
\hline Manufacture Costs & 274567018 & 199354647 & 1046262 & 1433535208 \\
\hline Employment Costs & 10247 & 16922 & 5232 & 50398 \\
\hline Other Costs & 66722064 & 37718679 & 83554 & 430050793 \\
\hline Energy Costs & 31806986 & 17968766 & 22103 & 150073357 \\
\hline Production Revenues & 502909093 & 299999017 & 2392067 & 3060606277 \\
\hline Other Revenues & 38594206 & 25620039 & 44402 & 153839171 \\
\hline The Value of the Exports & 166088319 & 99561207 & 15572 & 655721043 \\
\hline
\end{tabular}

As observed in Table 1, the variable values consist of quite high values. A separate model for each DMU was established and 54 models for deterministic DEA and one distinct $\alpha$ (alpha) level were processed for solving, and the obtained efficiency levels are shown as Objective Value in Table 2.

Table 2. Objective values for deterministic DEA and CCDEA for $\alpha=0.05$

\begin{tabular}{|c|c|c|c|c|c|c|c|c|}
\hline \multirow{2}{*}{ 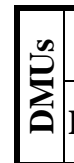 } & \multicolumn{2}{|c|}{ Objective Value } & \multirow{2}{*}{$\sum_{\theta}^{n}$} & \multicolumn{2}{|c|}{ Objective Value } & \multirow{2}{*}{$\sum_{0}^{n}$} & \multicolumn{2}{|c|}{ Objective Value } \\
\hline & Deterministic & $\begin{array}{l}\text { Stochastic } \\
\alpha=0.05\end{array}$ & & Deterministic & $\begin{array}{l}\text { Stochastic } \\
\alpha=0.05\end{array}$ & & Deterministic & $\begin{array}{l}\text { Stochastic } \\
\alpha=0.05\end{array}$ \\
\hline 1 & 0 & 0 & 19 & 0 & 0 & 37 & 0 & 0 \\
\hline 2 & 6.1458 & 807.9341 & 20 & 0 & 0 & 38 & 8.9286 & 1.0000 \\
\hline 3 & 16.3768 & 561.5519 & 21 & 0 & 0 & 39 & 33.5587 & 5.0000 \\
\hline 4 & 0 & 0 & 22 & 0 & 0 & 40 & 25.7650 & 1.0000 \\
\hline 5 & 147.3814 & 2.0000 & 23 & 5.5813 & 512.4047 & 41 & 0 & 0 \\
\hline 6 & 0 & 0 & 24 & 0 & 0 & 42 & 0 & 0 \\
\hline 7 & 15.3646 & 73.8221 & 25 & 805.3638 & 0 & 43 & 0 & 0 \\
\hline 8 & 0 & 0 & 26 & 32.6126 & 1.0000 & 44 & 0 & 0 \\
\hline 9 & 0 & 0 & 27 & 9.9581 & 347.3217 & 45 & 0 & 0 \\
\hline 10 & 0 & 0 & 28 & 4.6202 & 7.0000 & 46 & 1635.4305 & 1.0000 \\
\hline 11 & 0 & 0 & 29 & 473.3923 & 0 & 47 & 3.71906 & 0 \\
\hline 12 & 0 & 0 & 30 & 0 & 0 & 48 & 24.7622 & 0 \\
\hline
\end{tabular}


Table 2. (continued) Objective values for deterministic DEA and CCDEA for $\alpha=0.05$

\begin{tabular}{|l|l|l|l|l|l|l|l|l|}
\hline 13 & 41.3350 & 103.8449 & 31 & 4.7068 & 2.0000 & 49 & 0 & 0 \\
\hline 14 & 0 & 0 & 32 & 0 & 0 & 50 & 0 & 0 \\
\hline 15 & 0 & 0 & 33 & 0 & 0 & 51 & 0 & 0 \\
\hline 16 & 94.4264 & 168.2929 & 34 & 0 & 0 & 52 & 0 & 0 \\
\hline 17 & 9.7345 & 5.0000 & 35 & 0 & 0 & 53 & 0 & 0 \\
\hline 18 & 11.0523 & 231.0081 & 36 & 7.4046 & 841.4472 & 54 & 0 & 0 \\
\hline
\end{tabular}

Table 2 can be summarized as Table 3 .

Table 3. The number of efficient DMUs

\begin{tabular}{|l|c|}
\hline$\alpha$ level & The Number of Efficient DMUs \\
\hline Deterministic & 32 \\
\hline$\alpha=0.05$ & 36 \\
\hline
\end{tabular}

32 DMUs are efficient in deterministic DEA where the number of efficient DMUs tend to increase in stochastic process for $\alpha=0.05$. As it is seen from Table 3, 36 DMUs are efficient in CCDEA.

Table 4 shows the number of inefficient DMUs in terms of variables for deterministic DEA and CCDEA for $\alpha=0.05$.

Table 4. Number of inefficient DMUs by variable

\begin{tabular}{|c|c|c|c|c|c|c|c|}
\hline & \multicolumn{4}{|c|}{ Input variables } & \multicolumn{3}{|c|}{ Output Variables } \\
\hline & 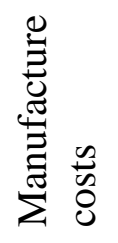 & 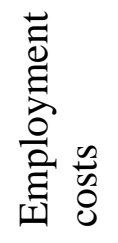 & 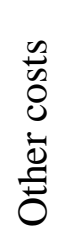 & 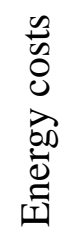 & 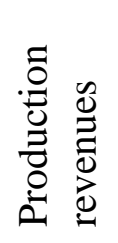 & 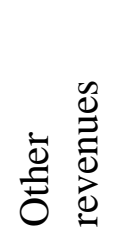 & 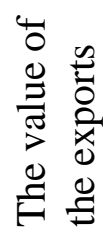 \\
\hline Deterministic & 5 & 12 & 7 & 5 & 17 & 12 & 18 \\
\hline$\alpha=0.05$ & 1 & 16 & 8 & 15 & 15 & 2 & 8 \\
\hline
\end{tabular}

Table 4 shows that in deterministic analysis only 5 DMUs have excess manufacture costs, 7 have excess other costs, and 5 have excess energy costs. Employment costs is the variable that creates the most inefficiency with 12 DMUs among input variables. The output variables indicate that a lot of DMUs are inefficient.

In terms of the input variables of CCDEA only 1 DMU has excess manufacture costs, 16 have excess employment costs, 7 have excess other costs, and 15 have excess energy costs. The output variables of the CCDEA shows that 15 DMUs have excess 
production revenues, 2 DMUs have excess other revenues, and 8 DMUs have excess value of exports.

When CCDEA and deterministic DEA results are compared it is observed that number of inefficient DMUs in input variables increase except manufacture cost, whereas number of inefficient DMUs in output variables decrease. Especially the inefficiency in other revenues and the value of exports variables are reduced drastically.

\section{CONCLUSION}

Compared with the deterministic process, number of inefficient DMUs decrease in all output variables when $\alpha=0,05$ although inefficient DMUs differ in input variables. The reduction in the number of inefficient outputs effect the result in a pozitive way despite inefficiency in input variables increase in the results of CCDEA.

The number of efficient DMUs increase from 32 to 36 when CCDEA $(\alpha=0,05)$ is compared to deterministic analysis as it is observed from Table 3. 32 efficient DMUs in deterministic analysis conserve their efficiency, and 4 more DMUs become efficient in CCDEA when $\alpha=0,05$ as it can be concluded from Table 2 . The increase in the number of efficient DMUs is mainly due to the reduction in the number of inefficient output variables. As a conclusion it can be said that the inefficient DMUs are so close to the efficiency frontier, so that $5 \%$ increase in outputs and $5 \%$ decrease in inputs will lead to efficiency.

The efficient DMUs determined by considering deterministic analysis show constrained BCC-additive model should be performed in the efficiency analysis for comparing the efficiencies of DMUs within the sector and developing production plans for the future as the inputs and outputs may change together.

\section{REFERENCES}

1. M.J. Farrell, The measurement of productive efficiency. Journal of the Royal Statistical Society, 120(3), 253-281, 1957.

2. W.H. Greene, Frontier Production Functions, in: Pesaran MH, Wickens MR (eds). Blackwell Publishers, London, 1997.

3. A. Charnes, W.W. Cooper and E. Rhodes, Measuring the efficiency of decisionmaking units. European Journal of Operational Research 2, 429-444, 1978.

4. R.D. Banker, A. Charnes and W.W. Cooper, Some models for estimating technical and scale efficiencies in data envelopment analysis, Management Science 30(9), 1078$1092,1984$.

5. A. Charnes, W.W. Cooper, B. Golany, L.M. Seiford and J. Stutz, Foundations of data envelopment analysis for Pareto-Koopmans efficient empirical production functions, Journal of Econometrics 30(1-2), 91-107, 1985.

6. C.A.K. Lovell, Linear programming approaches to the measurement and analysis of productive efficiencies, TOP 2(2), 175-243, 1994.

7. R.D. Banker and W.W. Cooper, Validation and generalization of dea and its uses, in TOP the new Journal of the Spanish Society of Statistics and Operations Research 2(2), 249-314, 1994. 
8. W.W. Cooper, Z. Huang, V. Lelas, S. Li and O.B. Olesen, Chance constrained programming formulations for stochastic characterizations of efficiency and dominance in DEA. Journal of Productivity Analysis 9(1), 53-80, 1998.

9. A. Charnes, W.W. Cooper, A.Y. Lewin and L.M. Seiford, Data envelopment analysis: Theory, methodology and applications, Kluwer Academic Publishers, Boston, 1994.

10. K.C. Land, C.A.K. Lovell and S. Thore, Chance-constrained data envelopment analysis. Paper presented at the National Science Foundation Conference, Charpel Hill, 87-98, 1988.

11. J.K. Sengupta, Data envelopment analysis for efficiency measurement in the stochastic case, Computers \& Operations Research 14, 117-129, 1987.

12. J.K. Sengupta, Robust efficiency measures in a stochastic efficiency model, International Journal of Systems Science 19, 779-791, 1988b.

13. J.K. Sengupta, Measuring economic efficiency with stochastic input-output data, International Journal of Systems Science 20(2), 203-213, 1989.

14. K.C. Land, C.A.K. Lovell and S. Thore, Chance-constrained data envelopment analysis, Managerial and decision economics 14(6), 541-554, 1993.

15. K.C. Land, C.A.K. Lovell and S. Thore, Productive Efficiency under Capitalism and State Socialism: An Empirical Inquiry Using Chance-Constrained Data Envelopment Analysis. Technological Forecast SocialChange 46, 139-152, 1994.

16. O.B. Olesen and N.C. Petersen, Chance Constrained efficiency evaluation. Management Science 41, 442-457, 1995.

17. O.B. Olesen, Comparing and combining two approaches for Chance constrained DEA, Journal of Productivity Analysis 26, 103-119, 2006.

18. W.W. Cooper, Z. Huang and S.X. Li, Satisficing DEA models under chance constraints, Annals of Operations Research, 66, 279-295, 1996.

19. S.X. Li, Stochastic models and variable return to scale in data envelopment analysis. European Journal of Operational Research 104, 532-548, 1998.

20. A. Desai, J.S. Ratick and A.P. Schinnar, Data envelopment analysis with stochastic variations in data. Socio-Economic Planning Sciences 39, 147-164, 2005. 